\title{
Monitoring of yeast cell concentration using a micromachined impedance sensor
}

\author{
E.E. Krommenhoek ${ }^{\mathrm{a}, *}$, J.G.E. Gardeniers ${ }^{\mathrm{a}}$, J.G. Bomer ${ }^{\mathrm{a}}$, A. Van den Berg ${ }^{\mathrm{a}}$, \\ X. Li ${ }^{\text {b }}$, M. Ottens ${ }^{\text {b }}$, L.A.M. van der Wielen ${ }^{\text {b }}$, G.W.K. van Dedem ${ }^{\text {b }}$, \\ M. Van Leeuwen ${ }^{\text {b }, ~ W . M . ~ v a n ~ G u l i k ~}{ }^{b}$, J.J. Heijnen ${ }^{b}$ \\ ${ }^{a}$ MESA+ Institute for Nanotechnology, University of Twente, P.O. Box 217, 7500 AE Enschede, The Netherlands \\ ${ }^{\mathrm{b}}$ Department of Biotechnology, Delft University of Technology, The Netherlands \\ Received 2 June 2005; received in revised form 19 September 2005; accepted 26 September 2005 \\ Available online 9 November 2005
}

\begin{abstract}
This paper describes the design, modeling and experimental characterization of a micromachined impedance sensor for on-line monitoring of the viable yeast cell concentration (biomass) in a miniaturized cell assay. Measurements in Saccharomyces cerevisiae cell culture show that the characteristic frequency describing the $\beta$-dispersion of $S$. cerevisiae cells is around $2.8 \mathrm{MHz}$. The permittivity change of the cell suspension was measured for the concentration range $0-9 \mathrm{~g} / \mathrm{l}$ and depends linearly on the biomass concentration. In order to compensate the measurements for the electric properties of the background electrolyte, which increases the sensitivity and allows measurements in different media, the use of a three-electrode configuration in combination with a semi-permeable poly(2-hydroxyethyl methacrylate) (pHEMA) membrane was explored. Measurements show that the impedance of hydrated pHEMA varies with the background electrolyte conductivity only, and not with the concentration of cells, indicating that pHEMA is suitable for this purpose. The optimal pHEMA membrane thickness was determined using finite-element modelling and was found to be $1 \mu \mathrm{m}$ for the electrode configuration under study.
\end{abstract}

(C) 2005 Elsevier B.V. All rights reserved.

Keywords: Dielectric spectroscopy; Permittivity; Cell concentration; Microporous membrane; pHEMA

\section{Introduction}

There is a growing interest in the miniaturization of cell cultivation systems, both for single-cell analysis [1] and for fermentation studies [2,3]. For the latter, monitoring of biomass with time is important for the assessment of the influence of fermentor conditions.

This paper will demonstrate that dielectric spectroscopy is a convenient method for the determination of biomass. In this method, the impedance of an electrochemical cell that contains the cell suspension is measured. The method is minimally invasive and selective for viable biomass only, as dead cells with leaky membranes do not affect the measurement. The design of the electrochemical cell requires only two metal electrodes with a defined spacing. Such a device can be fabricated using

\footnotetext{
* Corresponding author.

E-mail address: e.e.krommenhoek@utwente.nl (E.E. Krommenhoek).
}

standard thin-film processes and is cheap, small, scalable, steam-sterilizable and suitable for integration with other microfabricated electrochemical devices. The electrical equivalent circuit of the electrochemical cell and a typical impedance curve are shown in Fig. 1. The value of the electrolyte resistance $\left(R_{\mathrm{S}}\right)$ and the electrochemical cell capacitance $\left(C_{\mathrm{c}}\right)$ depend on the sensor dimensions and the conductivity and permittivity of the electrolyte, respectively.

The dielectric spectra of biological cells show three distinct dispersions in the frequency range of $1 \mathrm{~Hz}$ to $1 \mathrm{GHz}$, termed $\alpha-, \beta$ - and $\gamma$-dispersion [4]. $\beta$-Dispersion originates from interfacial polarization, that is the accumulation of charges at the boundaries between the membrane and aqueous phases of different electrical properties. This polarization is frequency dependent and shows relaxation [5]. The dispersive frequency range is represented by a characteristic frequency, which depends strongly on the exact morphology of the cells and the electric properties of the cells and the background electrolyte [6]. Previous studies showed characteristic frequencies in the 


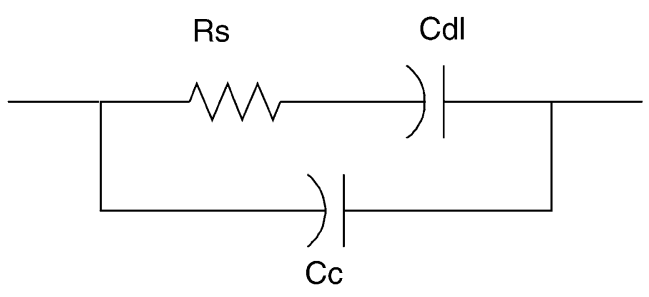

(a)

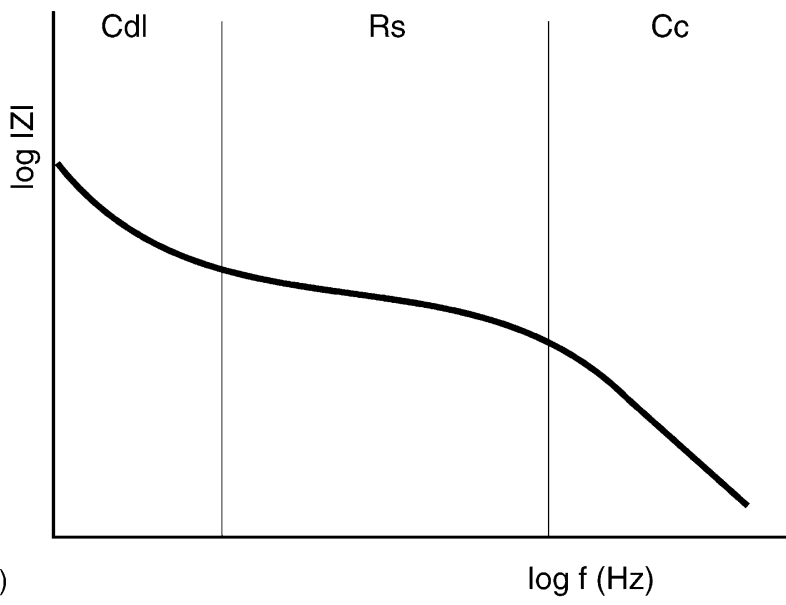

Fig. 1. (a) Electrical equivalent circuit and (b) typical impedance curve. $\mathrm{Cd}$ is the electrode double layer capacitance.

order of a few MHz for viable Saccharomyces cerevisiae cells [6,7]. Asami and Yonezawa [7] have described theoretically how $\beta$-dispersion affects the conductivity and permittivity of the cell suspension and how the cell concentration can be estimated from these data. In this work, the viable yeast cells are modeled as single-shell spherical cells. Our work relates this theory to experimental results obtained with a micromachined impedance sensor that was designed for the on-line monitoring of biomass in a miniaturized yeast assay. The sensor is also suitable for use in larger (up to industrial scale) fermentation systems.

The main reason that dielectric spectroscopy is still not a widely used method for biomass monitoring, is that the measured signals suffer from interference of the electric properties of the background electrolyte. The ionic concentration of the background electrolyte affects its conductivity. The measured permittivity is affected as well, as it determines the rate of electrode polarization. This effect is frequency dependent and cannot reliably be subtracted out, as the ionic content of the medium changes during the fermentation process [8]. Therefore, we have explored a method that compensates for changes in the electric properties of the background electrolyte by differential measurement using a three-electrode configuration [9] in which the impedance measured between two closely spaced electrodes is made insensitive to biomass by covering it with a porous membrane that prevents cells to approach the electrodes (Fig. 2).

The electric properties of a microporous poly(2-hydroxyethyl methacrylate) (pHEMA) membrane were characterized for this purpose. The advantages of pHEMA as membrane material are that it can easily be photostructured using standard photolitho-

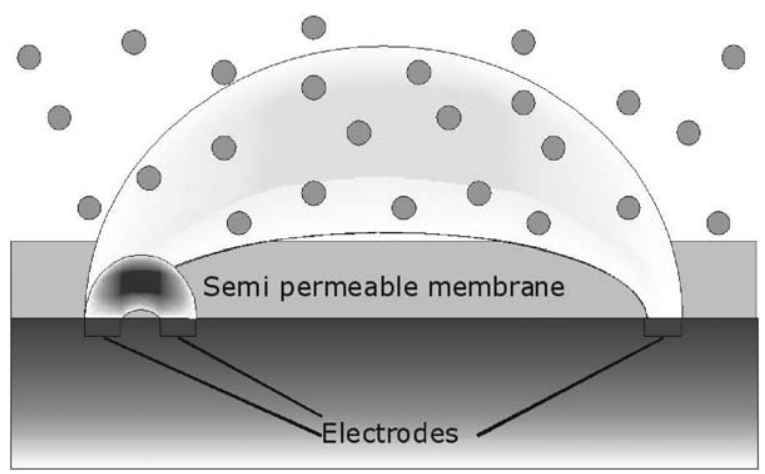

Fig. 2. Schematical representation of the three-electrode configuration.

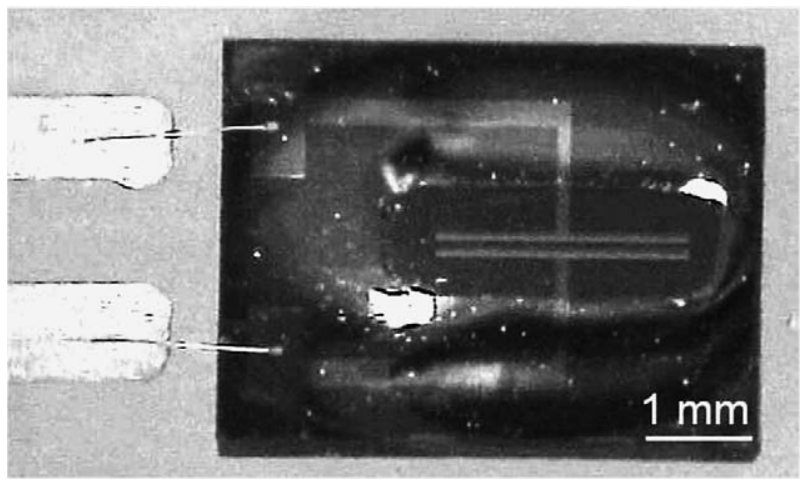

Fig. 3. Photograph of fabricated planar electrode structure.

graphic processes and that it withstands standard sterilization by autoclavation.

\section{Device fabrication}

Planar Ta/Pt electrodes were fabricated on an oxidized silicon substrate using a sputtering technique for metal deposition and lift-off photolithography. The processed electrode configuration consists of two $2200 \mu \mathrm{m} \times 100 \mu \mathrm{m}$ electrodes, spaced by $60 \mu \mathrm{m}$ (Fig. 3).

One pair of electrodes was covered by a pHEMA membrane. The HEMA mixture consists of $2.426 \mathrm{ml}$ HEMA, $0.092 \mathrm{ml}$ crosslinker (TEGDMA), 0.1538 gr. UV initiator (2,2dimetoxy-2-phenylacetophenone, DMPAP) and $1.169 \mathrm{ml}$ solvent (ethyleneglycol). The UV initiator is added at the end, in order to prevent the mixture from polymerizing too early.

The prepared solution is pipetted on the sensor, covered with a piece of Mylar foil and subsequently exposed to $366 \mathrm{~nm}$ UV light for $2 \mathrm{~min}$. The non-optimized membrane thickness of $300 \mu \mathrm{m}$ was high enough to assure that the electric field between the electrodes is concentrated within the hydrated pHEMA layer.

\section{Experimental}

The characteristic frequency for dielectric dispersion typically is around a few $\mathrm{MHz}$ for $S$. cerevisiae cells, but the exact frequency value depends on the morphology of the cells and the electrical properties of the medium. Therefore, the medium conductance in a standard yeast buffer in which $7 \mathrm{~g} / \mathrm{l} \mathrm{DW}$ of 
viable yeast cells were suspended, was measured as a function of frequency using a pair of bare $\mathrm{Ta} / \mathrm{Pt}$ electrodes and a Hewlett Packard HP 4194A gain-phase analyzer.

The theoretical response of the change in electrochemical cell capacitance due to dielectric dispersion to varying cell concentrations was calculated, based on the single-shell model for characterizing the suspended cells, the impedance model shown in Fig. 1(a), the conformal mapping technique [10] and the actual suspension parameters.

The relation between the change in electrochemical cell capacitance and the viable $S$. cerevisiae cell concentration was also measured. To this end, the yeast cells were suspended in a standard buffer solution, containing $10 \mathrm{~g} / \mathrm{l}$ ammonium sulphate, $6 \mathrm{~g} / \mathrm{l}$ potassium phosphate monobasic and $1 \mathrm{~g} / \mathrm{l}$ magnesium sulphate heptahydrate. The $\mathrm{pH}$ of the buffer solution was adjusted to 5.0 using concentrated sodium hydroxide. The applied voltage was kept below $100 \mathrm{mV}_{\text {rms }}$ in order to prevent redox reactions to take place.

Using the pHEMA-covered pair of electrodes, the electrolyte conductivity was measured in solutions containing known concentrations of sodium chloride. The measurements were compared to the theoretical electrolyte conductivity values, calculated using:

$\sigma_{\mathrm{c}}=\sum_{i} z_{i} c_{i} \lambda_{i}$

where $\sigma_{\mathrm{c}}$ is the electrolyte conductivity and $z_{i}$ and $c_{i}$ are the species valence and molar concentration of ion $i$, respectively. $\lambda_{i}$ is a parameter that describes the concentration dependence of the conductivities at $25^{\circ} \mathrm{C}$ [11]. The sensor response was also measured in cell suspensions of different biomass concentrations in order to verify whether the membrane is impermeable to yeast cells.

\section{Results and discussion}

First, the frequency range in which dielectric dispersion occurs was determined by measuring the impedance spectrum in a $7 \mathrm{~g} / \mathrm{l} \mathrm{DW}$ yeast cell suspension with a pair of bare $\mathrm{Ta} / \mathrm{Pt}$ electrodes. The difference between the conductance at a specific frequency and the conductance at low frequency in medium is plotted in Fig. 4.

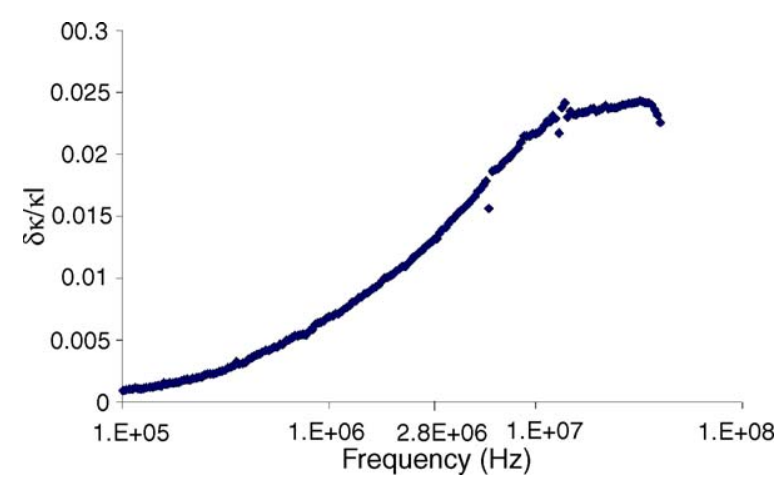

Fig. 4. Change in electrolyte conductivity in response to $7 \mathrm{~g} / \mathrm{l} \mathrm{DW}$ of yeast vs. frequency.

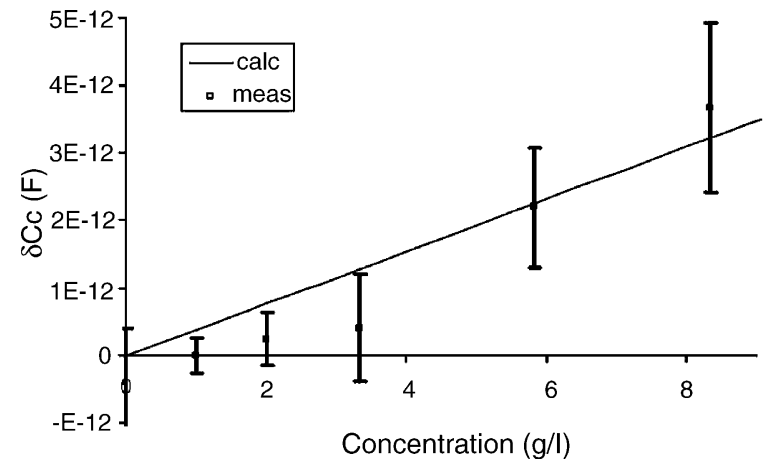

Fig. 5. Theoretical and measured response in yeast suspensions of various concentrations.

We see that the characteristic frequency describing this dispersion effect is around $2.8 \mathrm{MHz}$; at lower frequencies the effect decays to zero.

The electrochemical cell capacitance was measured in two different frequency ranges; the low-frequency limit was determined in the range of $60-80 \mathrm{kHz}$ and the dispersive value was determined in the range of $1-2 \mathrm{MHz}$. The measured difference between these values, $\delta C_{\mathrm{c}}$, shows a linear response to concentration and matches the calculations quite well for typical fermentor concentrations of yeast cells (Fig. 5) in a standard yeast buffer solution. The sensor performance depends to a large extent on the match between the sensor dimensions and the electric properties of the yeast suspension. The measurements show that a detection limit below $1 \mathrm{~g} / \mathrm{l}$ can be achieved once the sensor dimensions meet the conductivity of the liquid.

After placement in solution, the electrolyte resistance measured with the pHEMA covered electrode pair keeps decreasing for $40 \mathrm{~min}$ and then reaches a stable value. This can be seen in Fig. 6. It is assumed that this is due to hydration of the membrane. In future configurations, the electrodes will be much more closely spaced and the membrane thickness required to assure that the electrical field between the electrodes is concentrated within the hydrated membrane layer will be much lower. This will decrease the time required for membrane hydration. The time might be reduced even further by adding solvent to the HEMA mixture during fabrication, resulting in an opener structure.

The results in Fig. 7 show that the resistivity measured with a pHEMA covered conductivity sensor in $1-100 \mathrm{mM} \mathrm{NaCl}$ solutions is higher than the theoretical and experimentally obtained response of an uncovered sensor with a constant factor, indicating that the conductance measured between the electrodes indeed arises from the electrolyte conductance, but is lowered by the insulating membrane.

Fig. 8 shows the difference in cell capacitance, $\delta C_{\mathrm{c}}$, in two different frequency ranges $(60-80 \mathrm{kHz}$ and $1-2 \mathrm{MHz})$, measured with the pHEMA covered sensor as a function of the viable yeast concentration. There is no clear linear relation between the permittivity change and the viable yeast concentration, indicating that the membrane is indeed impermeable to yeast cells.

To find the optimal pHEMA thickness, the electrical field distribution in this system was simulated using finite-element 

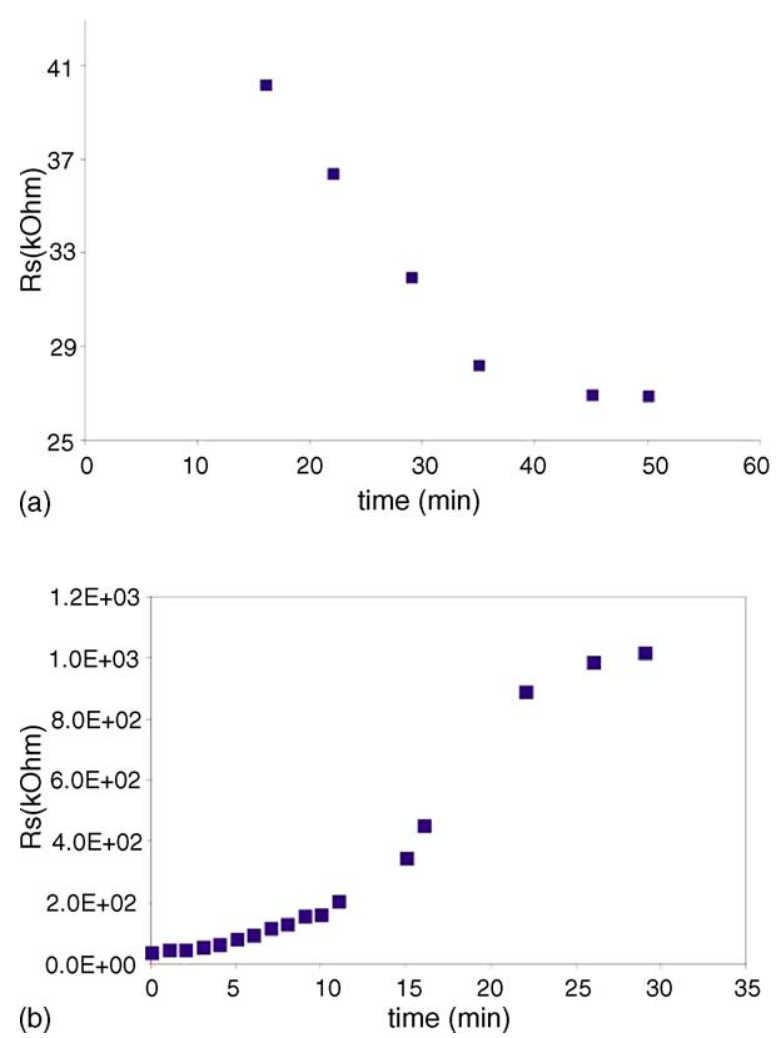

Fig. 6. Electrolyte resistance versus time after (a) placing in solution (b) changing the ionic concentration from 100 to $2 \mathrm{mM}$.
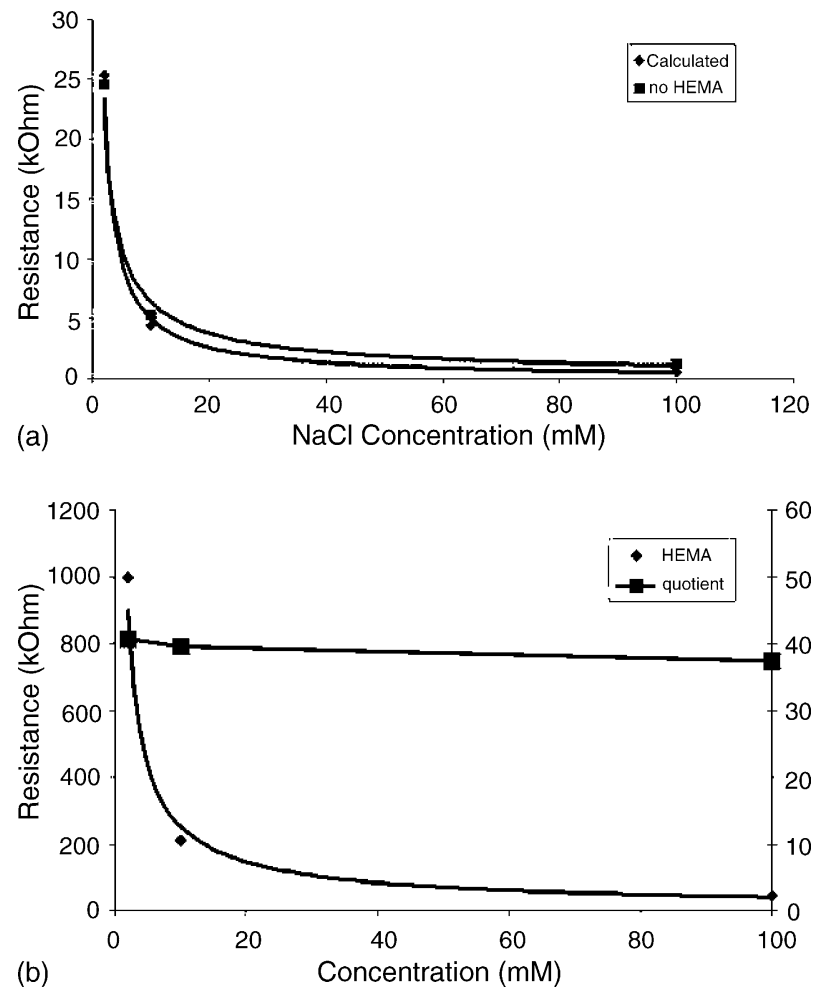

Fig. 7. Theoretical electrolyte resistance and measurements with (a) a planar conductance sensor and (b) a planar conductance sensor covered with a pHEMA membrane. The quotient plotted in (b) shows the relation between the resistances measured with both sensors is constant.

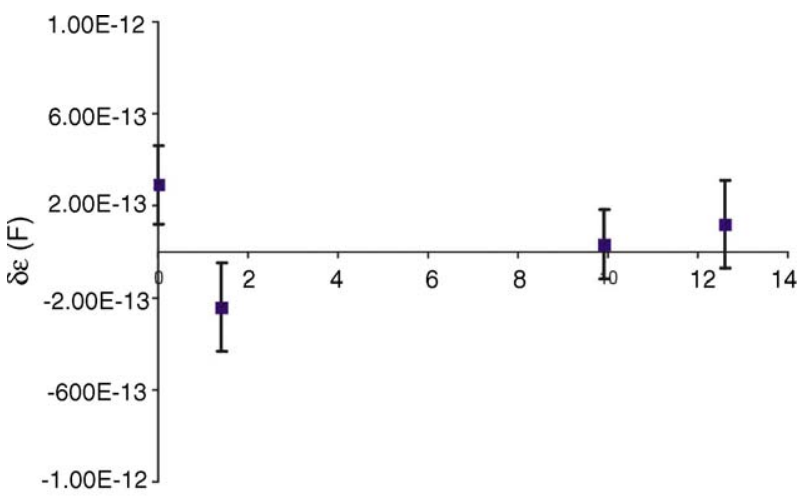

Concentration (g/l DW)

Fig. 8. Measured permittivity response of the pHEMA-covered impedance sensor in yeast suspensions of various concentrations.

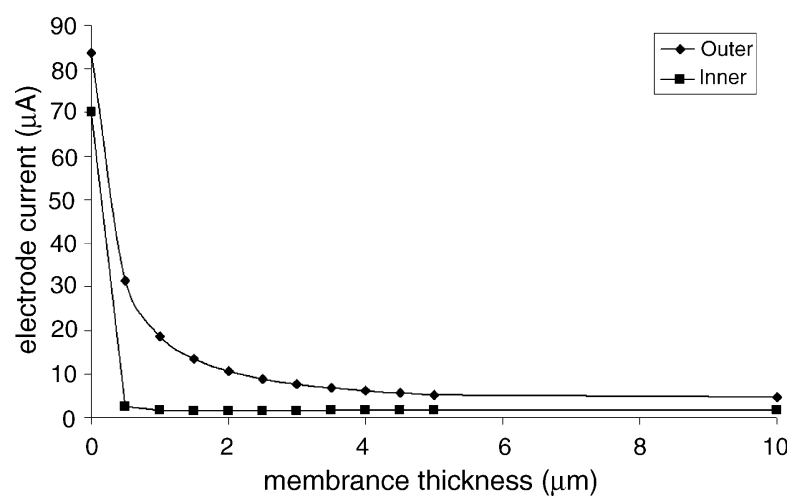

Fig. 9. Simulated relation between electrode current and membrane thickness.

modelling. The width of all electrodes was defined as $200 \mu \mathrm{m}$, the length of the outer electrodes is set to $200 \mu \mathrm{m}$ and the spacing between them to $400 \mu \mathrm{m}$. The length of the inner electrode is set to $2 \mu \mathrm{m}$ and the spacing between the two most closely spaced electrodes is set to $2 \mu \mathrm{m}$ as well. The electrodes are covered by a layer of varying thickness, which is assigned to the measured electric properties of the hydrated pHEMA membrane. The volume outside this layer is assigned to the dielectric properties of a cell suspension.

The current through the electrodes decreases with increasing membrane thickness (Fig. 9), as electrolyte conductivity decreases. The current through the inner electrodes reaches a steady value at a thickness of $1 \mu \mathrm{m}$, indicating that for this thickness the electrical field lines do not penetrate the cell-containing solution so that the measured signal does not depend on the cell concentration.

Future sensor designs will be based on the optimal pHEMA thickness resulting from these finite-element simulations.

\section{Conclusion}

Using a micromachined impedance sensor, the characteristic frequency describing the dielectric dispersion in a S. cerevisiae cell suspension was determined to equal $2.8 \mathrm{MHz}$. This value corresponds well to empiric data in literature. The measured capacitance change due to dielectric dispersion shows a linear 
response to concentration and matches the calculations quite well for typical fermentor concentrations of yeast cells in a standard yeast buffer solution, confirming the relevance of the applied theory on microreactor scale.

The measurements with the pHEMA covered electrode pair show that the measured resistance does depend linearly on the actual electrolyte conductivity and that the measured cell capacitance is independent of the viable yeast cell concentration. This confirms that the pHEMA membrane gets hydrated, but is impermeable to yeast cells. This makes the proposed three-electrode configuration suitable for determining the background electrolyte conductivity independently of the viable cell concentration. This signal can thus be used for compensating the measured impedance spectrum for changes in the background electrolyte.

The optimal hydrated membrane thickness arises from the observed electric properties of the membrane and finite-element modelling and is found to equal $1 \mu \mathrm{m}$ for the electrode configuration under study. The electric and transient properties of the membrane can be further optimized by altering the physical membrane properties.

\section{Future plans}

Integrated sensor designs, which integrate the proposed conductance sensor with other electrochemical sensors required for the online monitoring of fermentor conditions, are being processed at the cleanroom facilities of MESA+. The sensors will be tested in a fed-batch process and finally be integrated in a microscale fed-batch fermentor. There will be a focus on optimizing and processing the pHEMA membrane in a reproducible way.

\section{Acknowledgements}

Sebastiaan Herber is acknowledged for his help in hydrogel synthesis. This work is part of the ACTS-IBOS programme (IBOS 053.63.009), which is funded by the Dutch Science Foundation (NWO), DSM Anti-Infectives BV, Diosynth/Organon and Applikon BV.

\section{References}

[1] S. Gawad, K. Cheung, U. Seger, A. Bertsch, P. Renaud, Dielectric spectroscopy in a micromachined flow cytometer: theoretical and practical considerations, Lab Chip 4 (2004) 241-251.

[2] N. Szita, A. Zanzotto, P. Boccazzi, A.J. Sinskey, M.A. Schmidt, K.F. Jensen, Monitoring of cell growth, oxygen and $\mathrm{pH}$ in microfermentors, in: Proceedings of the $\mu$ TAS 2002 symposium, pp. 7-9.

[3] M. Maharbiz, W.J. Holtz, S. Sharifzadeh, J.D. Keasling, R.T. Howe, A microfabricated electrochemical oxygen generator for high-density cell culture arrays, J. Microelectromech. Syst. 12 (2003) 590-599.

[4] K. Asami, Characterization of biological cells by dielectric spectroscopy, J. Non-Cryst. Solids 305 (2002) 268-277.

[5] H.P. Schwan, Electrical properties of tissues and cell suspensions, Adv. Biol. Med. Phys. 5 (1957) 147.

[6] S. Hauttmann, J. Müller, In-situ biomass characterisation by impedance spectroscopy using a full-bridge circuit, Bioprocess Biosyst. Eng. 24 (2001) 137-141.
[7] K. Asami, T. Yonezawa, Dielectric analysis of yeast cell growth, Biochim. Biophys. Acta 1245 (1995) 99-105.

[8] S.A. Siano, Biomass measurement by inductive permittivity, Biotechnol. Bioeng. 55 (1997) 289-304.

[9] A.R. Varlan, P. Jacobs, W. Sansen, New design technique for planar conductometric haematocrit sensors, Sens. Actuators B 34 (1996) 258264.

[10] W. Olthuis, W. Steekstra, P. Bergveld, Theoretical and experimental determination of cell constants of planar-interdigitated electrolyte conductivity sensors, Sens. Actuators B 24-25 (1995) 252256.

[11] J.O'M. Bockris, A.K.N. Reddy, Modern Electrochemistry, vol. 1, Section 4.4, Plenum Press, New York, 1970.

\section{Biographies}

Erik Krommenhoek was born in Apeldoorn, the Netherlands, on July 29, 1979. He received the MSc degree in Electrical Engineering from the University of Twente, Enschede, the Netherlands, in 2004 on the flow dependence of interdigitated conductivity sensors. He is now working as a $\mathrm{PhD}$ student at the Bios, Lab-on-a-chip Group at the faculty of Electrical Engineering, Mathematics and Computer Science at the same university. The subject of the $\mathrm{PhD}$ project is the development of miniaturized fed-batch fermentors for high throughput screening of cells.

J.G.E. ("Han") Gardeniers (BSc: 1982; MSc: 1985; PhD: 1990) joined the Department of Electrical Engineering at the University of Twente, The Netherlands, as an assistant professor in 1990. From 2001 till 2003 he was employed as project leader MOEMS at Kymata Netherlands B.V./Alcatel Optronics Netherlands B.V. and as research manager at Micronit Microfluidics B.V., where he was responsible for $R \& D$ related to miniaturized chemical synthesis and analysis systems. Since January 2003, he has an associate professor position with the Department of Electrical Engineering, Mathematics and Informatics at the University of Twente, The Netherlands, with the Biosensors/Lab-on-a-Chip Group. He has published over 70 reviewed journal papers on various topics in materials science, Microfabrication and Microfluidics.

Johan Bomer was born in Eibergen, The Netherlands, on September 6, 1958. He received the BS degree in applied physics from the Hoger Technische School, Enschede, The Netherlands, in 1981. From 1983 until 1986, he worked as technologist in the Semiconductor Physics Group of the University of Groningen. Since 1986 he works as process engineer in the Bios-Lab on a chip group (former Biosensor Technology Group), part of the MESA+ Research Institute, of the University of Twente.

Albert van den Berg received his Masters degree in applied physics from the University of Twente, The Netherlands in 1983. In 1988 he finished his thesis at the same university on the topic of chemically modified ISFETs. From 1988 to 1990 he was at the Swiss Center for Microelectronics and Microtechnology (CSEM) in Neuchâtel, Switzerland, project manager in the Chemical Sensor Department. From 1990-1993 he did research on miniaturized chemical sensors and sensor systems at the IMT, University of Neuchâtel, Switzerland. From 1993 until 1999 he was research coordinator Micro Total Analysis Systems ( $\mu$ TAS) at MESA, University of Twente, later extended to Miniaturized Chemical Systems (MiCS). In 1998 he was appointed as full professor in the Lab-on-a-Chip group. Albert van den Berg is and has been member of numerous scientific committees ( $\mu$ TAS, MEMS, Micromachine Summit, Transducers, Nanotech) and served as editor of the section $\mu$ TAS of Sensors and Actuators B. In 2002 he received the "Simon Stevin Master" award from the Dutch Technical Science foundation. He has published over 100 peer reviewed papers and edited several books on Labs-on-a-Chip. His current research interests focus on analysis systems and sensors, microreactors, micro- and nanofluidics and Lab-in-a-Cell.

Xiaonan Li was born in Beijing, China, on Oct. 31, 1977. He received the bachelor degree in chemistry from Beijing University, Beijing, China, in 2000. Then he received the MSc degree in Biochemical Engineering from the Technical University of Delft, Delft, the Netherlands, in 2002. He is now 
working as a PhD student at the Apply Physics Department, Bio- Separation Technology (BPT) group in TU Delft. The subject of the PhD-project is the development of miniaturized fed-batch fermentors for high throughput screening of cells.

Marcel Ottens received his MS in ChemEng in 1990 from Groningen University, his PDEng in Process Design in 1993 from Twente University of Technology, and his PhD in ChemEng in 1998 from Amsterdam University (the Netherlands). He is currently assistant professor Bioseparation Technology at the Delft University of Technology where he has been since 1998. His current research interests are crystallization and precipitation of biomaterials, fast conceptual bioprocess design, process chromatography (Simulated Moving Bed technology), bioprocess equipment miniaturization, micro systems technology and more in general transport phenomena and bio separation science. He published over 30 papers and several patents, presented dozens of (invited) lectures. He is board member of the Dutch Biotechnological Society, working party Product Isolation and Purification and member of the ACS, EFB, KNCV, NPT and NBV.

Luuk A.M. van der Wielen hold a MSc degree in Chemical Engineering from Twente University (Netherlands), and a PhD degree from Delft University of Technology. Currently, he is Full Professor at the Department of Biotechnology at the Delft University of Technology, the Netherlands where he heads the Bioseparations Section effectively since 1998. The activities of the section were ranked as excellent by the national research evaluation by the VSNU, and have resulted in several spin-off companies. He is the scientific director of B-BASIC, which is a focused program within NWOACTS. In addition, he is Visiting Professor at the University of San Carlos, Cebu City, the Philippines. Luuk van der Wielen is member of the editorial and advisory boards of several leading international scientific journals, and chaired several scientific conferences (ESBES4, BPP2005). He is a member of several national and European committees, as well as advisor to several national and international industries. $\mathrm{He}$ is one of the initiators of the successful academic program on Life Science and Technology of the Leiden University and TU Delft, and the director of the postgraduate program for Bioprocess Designers.
Gijs van Dedem received his Masters degree in food engineering from the University of Wageningen, The Netherlands in 1968. From 1968 to 1971 he was involved in the development of fermentation processes at Unilever Research Laboratory, The Netherlands. He received his $\mathrm{PhD}$ degree in biochemical engineering at the University of Waterloo Ontario, Canada in 1974. From 1974 to 2005 he worked on the development of isolation and purification processes for bulk biopharmaceuticals and analytical methods for products and impurities, validation and equipment qualification at Diosynth, The Netherlands. He joined the department of analytical biotechnology at Delft University of Technology, The Netherlands, as part-time full professor in 1996. He published over 60 reviewed journal papers on biotechnology and biochemistry.

Michiel van Leeuwen obtained his MSc degree in bioproces technology at the Wageningen University in 1999. He obtained a second MSc degree in philosophy at the free university of Amsterdam in 2002. Thereafter he worked at the public relations department of the Amsterdam University. In September 2003 Michiel started as a PhD student at the Department of Biotechnology at the Delft University of Technology. His research subject is the development of micro bioreactors.

W.M. ("Walter") van Gulik obtained his MSc degree in biology at the Leiden University in 1985. He obtained his PhD at the Delft University of Technology, Dept. of Bioprocess Technology in 1989. Thereafter he worked as a postdoc on several projects, both at the Delft University and at the Ecole Polytechnique Fédérale de Lausanne in Switzerland. Main areas of research were modeling of growth and product formation in fermentor cultivated microbial, plant and animal cells, biocalorimetry and metabolic flux analysis. In April 2000 he became assistant professor at the Delft University in the field of modeling and metabolic engineering of industrial microorganisms.

J.J. Heijnen is since 1988 professor in Bioprocess technology at Delft University of Technology, The Netherlands, after 15-year industrial experience. His fields of interests are design of (micro) bioreactors, metabolic engineering, bio-thermodynamics, mathematical modelling of organism. 